\title{
Measurement of urinary mercury excretion by atomic absorption in health and disease
}

\author{
ANDREW TAYLOR and VINCENT MARKS \\ South West Metropolitan Region Heavy Metals Reference Centre, Department of \\ Biochemistry, University of Surrey, Guildford
}

\begin{abstract}
Taylor, A., and Marks, V. (1973). British Journal of Industrial Medicine, 30, 293-296. Measurement of urinary excretion by atomic absorption in health and disease. Excretion of mercury was measured by a cold-vapour atomic absorption technique on samples of urine from five groups of people having varying exposure to mercury.

Serial investigations of up to 14 days were carried out on eight subjects to determine the temporal relationship between exposure and excretion.

Subjects with no exposure excreted 0-10 $\mu$ g mercury per gramme creatinine. Similar values were found in laboratory staff and men assembling hollow cathode lamps. Excretion of mercury by dental workers was significantly increased. No correlation between exposure and excretion of mercury was seen in the subjects investigated.

The significance of measuring urinary excretion in the detection of mercury intoxication is discussed. The suggestion is made that urinary mercury excretion of more than $20 \mu \mathrm{g} / \mathrm{g}$ creatinine or $40 \mu \mathrm{g}$ mercury per 24 hours should be considered evidence of recent or remote exposure to mercury. It is concluded that measurement of urinary mercury excretion is important in revealing those persons who may ultimately develop symptoms of toxicity.
\end{abstract}

Mercury and mercurial compounds are used in many industries and manufacturing processes and may, if improperly used or if hygienic conditions are not adequate, give rise to toxic symptoms and even permanent neurological damage. Neither the concentration of mercury in the blood nor its excretion in the urine correlates well with the appearance and severity of signs and symptoms of mercury intoxication in persons exposed to metallic mercury (Smith, Vorwald, Patil, and Mooney, 1970; Kark, Poskanzer, Bullock, and Boylen, 1971; Wallach, 1972). A better correlation can be shown between toxicity and the intensity of exposure to mercury vapour and its duration. Nevertheless, measurement of urinary mercury excretion remains the best test available for confirming a diagnosis of mercury intoxication in individual persons working with the metal who have suggestive symptoms (Goldwater, 1964).

In order to reveal excessive exposure it is essential Five groups of subjects were investigated: to know how much mercury is excreted by healthy persons who work continuously with mercury. Because of the introduction of new and more sensitive analytical techniques, values for urinary mercury reported in the literature are no longer applicable and may be misleading. We have, therefore, sought to determine the amount of mercury excreted by seemingly healthy people working with mercury in a variety of industries as well as in control non-exposed subjects. This could provide a basis for comparison with symptomatic subjects working in the same industry.

In some subjects urinary mercury excretion was measured on consecutive days to determine the temporal relationship between exposure and excretion.

\section{Materials and methods}




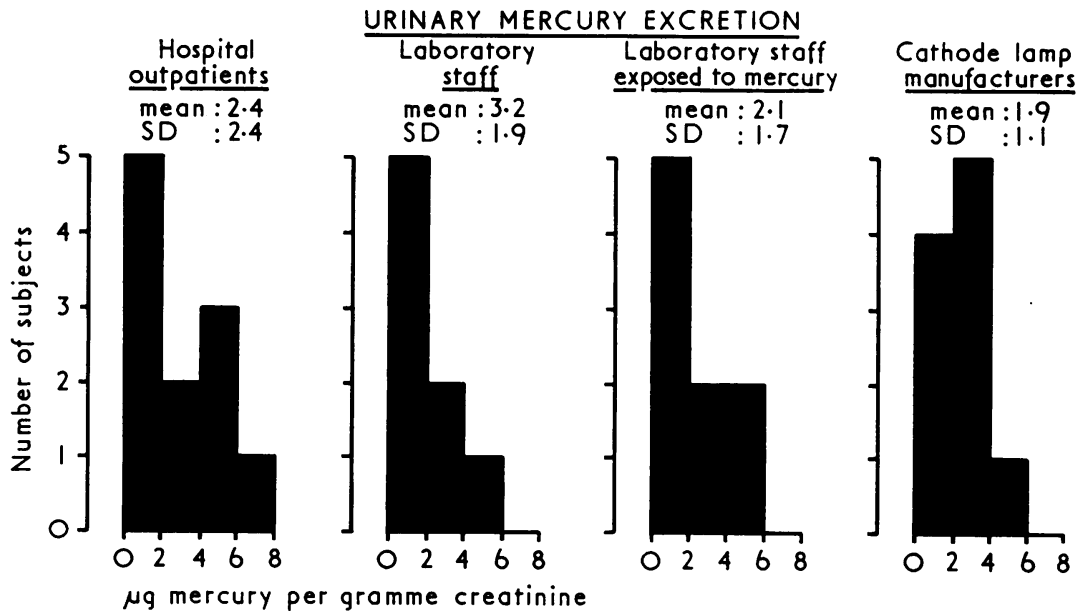

FIG. 1

(1) a random selection of hospital outpatients with no known exposure to mercury (11);

(2) hospital personnel with no known exposure to mercury (8);

(3) hospital and university workers using metallic mercury (9)

(4) assemblers of mercury hollow cathode lamps (10);

(5) dentists and dental surgery assistants (24).

Specimens of urine, preferably the first of the day, were collected into plastic universal containers (Sterilin Ltd) previously shown to be mercury free and not to adsorb mercury during urine storage (Taylor and Marks, 1973). Two subjects from group 3 and six from group 5 also took part in a serial investigation. Urine samples were collected at the same time each day for up to 14 days.

Urine samples were digested overnight with the sulphuric acid, permanganate, hydroxylamine hydrochloride reagent described by Lindstedt (1970). Mercury was determined using the cold-vapour apparatus described by Hatch and Ott (1968) on a Perkin Elmer model 303 atomic absorption spectrophotometer. In our hands this method gives an average recovery of $97 \%$ (mean of 35 determinations) at a urinary mercury concentration of approximately $100 \mu \mathrm{g} / \mathrm{l}$; a coefficient of variation for 'within batch' determinations of 5.2 at a mean urinary mercury concentration of $27.4 \mu \mathrm{g} / 1$; and of 8.1 for 'between batch' determinations at a mean urine concentration of $107 \mu \mathrm{g} / \mathrm{l}$. Results of the mercury analysis were expressed as $\mu \mathrm{g}$ mercury per gramme of urinary creatinine in order to minimize problems due to variations in urine osmolality and specific gravity.

\section{Results}

The concentration of mercury in the urine of people not exposed to mercury was $0-10 \mu \mathrm{g} / \mathrm{g}$ creatinine (roughly equivalent to $0-10 \mu \mathrm{g} / 1$ of urine). There was no difference in urinary output between laboratory workers and the general outpatient popu- lation. Excretion was not increased in laboratory staff working with metallic mercury. Similarly, urinary mercury excretion was indistinguishable from that of outpatient controls in men manufacturing hollow cathode lamps, all of whom had worked for at least 20 years in a room where mercury was in continuous use and sometimes left exposed to the atmosphere (Fig. 1). Only dental workers among the groups studied showed a significant $(P<0.001)$ increase in urinary mercury excretion above that of the controls (Fig. 2).

The urinary mercury excretion of a laboratory technician was measured each day for nine days during distillation of metallic mercury. There was no significant increase in urinary mercury during this period. A similar investigation was carried out during a week-long experiment in which a polarograph was used, and again no significant change in urinary mercury was detected.

\section{MERCURY EXCRETION IN 24 DENTAL WORKERS}

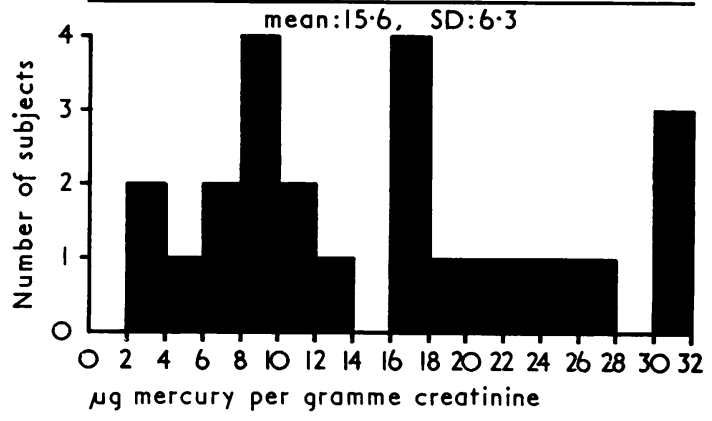

FIG. 2 
Six of the dental workers were investigated serially for daily urinary mercury excretion during two weeks. In only one subject was there any suggestion of a correlation between urinary mercury and the use of mercury during the preceding few days. Even here the association was not close. In the remaining five subjects there was no consistent change in daily urinary mercury excretion throughout the period of study.

\section{Discussion}

Excretion of mercury in the urine is believed (Goldwater, 1964) to be a good index of exposure to, and absorption of, metallic mercury. It probably reflects the care and precautions taken during its use.

Previous estimates of urinary mercury excretion by normal healthy subjects unexposed to metallic mercury are probably too high. Until recently the rnethods of measurement available were of limited specificity and sensitivity. Noe (1960), for example, considered an abnormal urinary mercury measurement as one in which the concentration exceeded $100 \mu \mathrm{g} / \mathrm{l}$. Nobel and Leifheit (1961), on the other hand, considered that the urinary mercury excretion should not ordinarily exceed $30 \mu \mathrm{g} / 1$ measured by the analytical techniques then available. Wallach (1972) suggested, without reference to the analytical methods employed, that urinary mercury concentrations are normally within the range $0-10 \mu \mathrm{g} / \mathrm{l}$. Battistone, Sammons, and Miller (1973), using an analytical technique for mercury similar to ours, found a mean mercury concentration of $8 \mu \mathrm{g} / \mathrm{l}$ in the urine of control subjects. A somewhat higher figure of $23 \mu \mathrm{g} / \mathrm{l}$ was reported by Howie and Smith (1967) using neutron activation. The median figure was only $13 \mu \mathrm{g} / \mathrm{l}$, however, suggesting that the results for the group as a whole were weighted by the inclusion of some subjects who had, at some time, unknowingly been exposed to metallic mercury.

Because urine osmolality or specific gravity can vary so much we suggest that comparison of results is made easier if mercury excretion is expressed as $\mu \mathrm{g}$ of mercury per gramme of creatinine excreted rather than merely as its urinary concentration. Our results and those of Battistone et al. (1973) suggest that urinary excretion exceeding $40 \mu \mathrm{g}$ per day ( $20 \mu \mathrm{g} / \mathrm{g}$ creatinine) should be taken as evidence of excessive but not necessarily harmful exposure to mercury.

Our data show that the potentially toxic procedure of mercury distillation can be carried out, providing stringent safety precautions are taken, without significant increase in urinary mercury excretion. This contrasts with the increased mercury excretion by the young female dental worker investigated by serial measurements who was relatively inexperienced and might, therefore, have been expected to be less careful when handling mercury amalgam than people unaware of its dangers.

Detailed information relating mercury excretion to signs of toxicity is still sorely lacking. In an extensive and detailed study of mercury excretion by workers in the chlorine manufacturing industry Smith and associates (1970) found that signs and symptoms attributable to mercury intoxication correlated poorly with urinary mercury but relatively well with the mercury content of the air and duration of exposure.

Two major clinical types of mercury intoxication are recognized, one due to inorganic mercury and the other to alkyl mercury compounds (Bidstrup, 1964) although this view has recently been challenged (Kark et al., 1971). Mercury toxicity of the first kind is rarely encountered nowadays in this country but was formerly quite common in people employed in certain manufacturing industries. In those cases in which urinary mercury was measured amounts greater than any found in the present survey and usually in excess of $1000 \mu \mathrm{g}$ per 24 hours were reported.

We have found that mercury excretion by dental workers was often abnormal when compared with that of the general population. The dental workers who were examined represented a cross section of the profession in respect of age and experience. All were healthy in so far as they were asymptomatic, which suggests that moderate exposure to mercury within this group, though more than within the general population, is not necessarily associated with harmful effects. Battistone et al. (1973) obtained results essentially similar to ours in 70 dental workers from six different clinics. Moreover, they found that the mean urinary mercury excretion correlated well with premises design and the level of mercury hygiene prevailing in the individual clinics. In the best clinics mean urinary mercury excretion was $13 \mu \mathrm{g} / 1$ compared with $56 \mu \mathrm{g} / 1$ in the worst and $8 \mu \mathrm{g} / \mathrm{l}$ in unexposed controls.

Our results, like those of Battistone et al. (1973), provide no information about the level of mercury excretion that can be taken as indicative of excessive or harmful exposure to mercury and the likelihood of mercury toxicity. The figure of $300 \mu \mathrm{g}$ mercury per litre of urine, suggested by Noe (1960), has received some acceptance (Gledhill and Hopkins, 1972; Smith et al., 1970) and, indeed, most cases of overt toxicity due to mercury reported in the literature have had concentrations exceeding this value (Kark et al., 1971; Gledhill and Hopkins, 1972). However, toxic symptoms undoubtedly occur in patients with urinary mercury excretions substantially below this figure. We have found, for example, that abnormal behaviour attributed by the factory doctor to mercury intoxication, in four men concerned in the manufacture of mercury ther- 
mometers, was associated with urinary mercury excretion rates within the range 46-67 $\mu \mathrm{g}$ per 24 hours. On the other hand, Wallach (1972) reported the case of a patient excreting more than $600 \mu \mathrm{g}$ mercury per day who showed no evidence of mercurial toxicity.

Modern sensitive analytical procedures make detection of increased exposure to mercury more readily amenable to investigation than before. Excretion of mercury in amounts exceeding that found in normal individuals, i.e., $20 \mu \mathrm{g} / \mathrm{g}$ creatinine, and, perhaps even more important, in the subject's own peer group should indicate the need-even in the absence of symptoms-for more extensive safety precautions and hygiene or even removal from exposure.

\section{References}

Battistone, G. C., Sammons, D. W., and Miller, R. A. (1973). Mercury excretion in military dental personnel. Oral Surgery, Oral Medicine and Oral Pathology, 35, 47-52.

Bidstrup, P. L. (1964). Toxicity of Mercury and its Compounds. Elsevier, Amsterdam.

Gledhill, R. F., and Hopkins, A. P.(1972). Chronic inorganic mercury poisoning treated with $\mathrm{N}$-acetyl-D-penicillamine. British Journal of Industrial Medicine, 29, 225-228.
Goldwater, L. J. (1964). Occupational exposure to mercury. Journal of the Royal Institute of Public Health and Hygiene, 27, 279-301.

Hatch, W. R., and Ott, W. L. (1968). Detection of submicrogram quantities of mercury by atomic absorption spectrophotometry. Analytical Chemistry, 40, 2085-2087.

Howie, R. A., and Smith, H. (1967). Mercury in human tissue. Journal of the Forensic Science Society, 7, 90-96.

Kark, R. A., Poskanzer, D. C., Bullock, J. D., and Boylen, G. (1971). Mercury poisoning and its treatment with N-acetyl-D,L,-penicillamine. New England Journal of Medicine, 285, 10-16.

Lindstedt, G. (1970). A rapid method for the determination of mercury in urine. Analyst, 95, 264-271.

Nobel, S., and Leifheit, H. C. (1961). Mercury in urine. In Standard Methods of Clinical Chemistry, Vol. 3, edited by D. Seligson, pp. 176-181. Academic Press, New York and London.

Noe, F. E. (1960). Chronic mercurial intoxication. Industrial Medicine and Surgery, 29, 559-564.

Smith, R. G., Vorwald, A. J., Patil, L. S., and Mooney, T. F. (1970). Effects of exposure to mercury in the manufacture of chlorine. American Industrial Hygiene Association Journal, 31, 687-700.

Taylor, A., and Marks, V. (1973). Contamination from syringes and blood container pots in trace element analysis. Annals of Clinical Biochemistry (In press).

Wallach, L. (1972). Aspiration of elemental mercuryEvidence of absorption without toxicity. New England Journal of Medicine, 287, 178-179.

Received for publication 29 November, 1972. Accepted for publication 4 April, 1973. 